High Speed Projectile Wounds: A Review of 22 Cases Dr. Savino Gasparini

Rio de Janeiro, BRAZIL

Introduction: With the rising incidence of urban violence related events, we observed a proportional rise in the incidence of victims of High-Speed-Projectile-caused wounds. Methods: This is a retrospective study of 22 cases of High Speed Projectile-caused wounds attended at the Miguel Couto Municipal Hospital Emergency Ward (Rio de Janeiro, Brazil) between March 1993 and June 1999.

Results: Eleven patients were dead on arrival, three presented with severe lesions and died before or during the surgical procedures, and eight patients were operated and survived.

Conclusion: Because of the high destructive power of this kind of projectiles, the majority of these patients do not survive to be treated (do not arrive to the hospital in time). Those who are submitted for surgical treatment usually have multiple associated lesions, requiring complex treatment and high skilled surgeons. This study shows the urgency of investigating and proposing new therapeutic procedures for the treatment of this kind of wounds, that are far more severe and lethal than are the ordinary gunshot wounds.

Key words: projectiles, high speed; mortality; surgery; violence; wounds

E-mail: lucanunes@dr.com

Prebosp Disast Med 2001;16(2):s31.

\section{Summary Gas Pressures in Plasma and Spinal Fluid} in "Normal" Humans

G.Y. Gebel; V.N. Utkin; A.N. Dasaev; L.M. Golostenova; N.G. Ignatov; S.G. Suvorov

"Medicine for You" Corporation, "VEZHA" ltd, Moscow, RUSSIA

Introduction: In Disaster Medicine, homeostasis of the respiratory gases takes part in the development of polyorganic pathology. Therefore it is desirable to study "normal" homeostasis.

Method: Tests on $\mathrm{pO}_{2}$ and $\mathrm{pCO}_{2}(\mathrm{mmHg}), \mathrm{SO}_{2}, \mathrm{pH}$, etc. and arterio-venous gradients $(\mathrm{D}(\mathrm{a}-\mathrm{v}))$ are used commonly to study human respiratory gas homeostasis. Beginning from 1987, we used tests on the summary gas pressure (SGP, $\mathrm{pS}$ in $\mathrm{mmHg}$ ) $\mathrm{pS}=\mathrm{pO}_{2}+\mathrm{pCO}_{2}$. The $\mathrm{D}(\mathrm{a}-\mathrm{v})$ on SGP are denoted as "gas functionals" $[\mathrm{DpS}=\mathrm{pSa}-\mathrm{pSv}]$ in $\mathrm{mmHg}$. SGP tests neither exclude nor substitute conventional $\left(\mathrm{pO}_{2}\right.$ and $\left.\mathrm{pCO}_{2}\right)$ tests, but include them as a part choosing only additive characteristic pressure. The knowledge of "normal" values for these tests are helpful for gas homeostasis. ${ }^{1}$ The investigation has been carried out during air respiration in supine position from the "normal" persons. Blood samples were taken from aortae (a), s. coronarius $(\mathrm{sC})$, bulbus v. jugularis int.(vJ), v. hepaticae (vH), a. pulmonalis $(\mathrm{aP})$, v. renalis $(\mathrm{vR})$, v. cava inf. $(\mathrm{vC})$, cerebrospinal fluid - liquor (L). Cases with $\mathrm{pO}_{2}=70-100$ and $\mathrm{pCO}_{2}=30-45 \mathrm{mmHg}$.

Results:

\begin{tabular}{lcrr} 
& $\mathbf{p O}_{2}$ & \multicolumn{1}{c}{$\mathbf{p C O}_{2}$} & pS \\
$\mathrm{a}$ & $91.7 \pm 0.54$ & $36.6 \pm 0.21$ & $128.3 \pm 0.60$ \\
$\mathrm{SC}$ & $23.8 \pm 0.30$ & $47.5 \pm 0.34$ & $71.3 \pm 0.60$ \\
$\mathrm{D}(\mathrm{a}-\mathrm{sC})$ & $67.9 \pm 0.43$ & $-10.9 \pm 0.26$ & $57.0 \pm 0.42$ \\
$\mathrm{a}$ & $85.7 \pm 0.65$ & $37.4 \pm 0.23$ & $123.1 \pm 0.67$ \\
$\mathrm{~V}$ & $38.0 \pm 0.57$ & $47.0 \pm 0.24$ & $84.9 \pm 0.54$ \\
$\mathrm{D}(\mathrm{a}-\mathrm{vJ})$ & $47.8 \pm 0.63$ & $-9.6 \pm 0.26$ & $38.2 \pm 0.58$ \\
$\mathrm{a}$ & $90.4 \pm 0.56$ & $39.4 \pm 0.31$ & $129.8 \pm 0.70$ \\
$\mathrm{vH}$ & $43.6 \pm 0.49$ & $44.9 \pm 0.34$ & $88.5 \pm 0.54$ \\
$\mathrm{D}(\mathrm{a}-\mathrm{vH})$ & $46.8 \pm 0.55$ & $-5.5 \pm 0.25$ & $41.3 \pm 0.60$ \\
$\mathrm{a}$ & $86.7 \pm 0.52$ & $37.6 \pm 0.26$ & $124.3 \pm 0.60$ \\
$\mathrm{aP}$ & $43.0 \pm 0.40$ & $41.9 \pm 0.30$ & $84.8 \pm 0.50$ \\
$\mathrm{D}(\mathrm{a}-\mathrm{aP})$ & $43.8 \pm 0.47$ & $-4.3 \pm 0.21$ & $39.5 \pm 0.45$ \\
$\mathrm{a}$ & $88.1 \pm 0.81$ & $36.5 \pm 0.45$ & $124.1 \pm 0.8$ \\
$\mathrm{vC}$ & $42.8 \pm 0.39$ & $45.3 \pm 0.42$ & $88.1 \pm 0.71$ \\
$\mathrm{D}(\mathrm{a}-\mathrm{aC})$ & $45.5 \pm 1.22$ & $-8.8 \pm 0.79$ & $36.7 \pm 1.21$ \\
$\mathrm{a}$ & $88.8 \pm 0.56$ & $38.4 \pm 0.23$ & $127.2 \pm 0.60$ \\
$\mathrm{vR}$ & $59.4 \pm 0.80$ & $41.4 \pm 0.24$ & $100.7 \pm 0,80$ \\
$\mathrm{D}(\mathrm{a}-\mathrm{vR})$ & $29.4 \pm 0.54$ & $-2.9 \pm 0.16$ & $26.5 \pm 0.50$ \\
$\mathrm{a}$ & $83.0 \pm 1.8$ & $38.8 \pm 0.56$ & $121.7 \pm 1.4$ \\
$\mathrm{~L}$ & $81.3 \pm 1.97$ & $46.3 \pm 0.76$ & $107.5 \pm 1.11$ \\
$\mathrm{D}(\mathrm{a}-\mathrm{L})$ & $21.7 \pm 0.22$ & $-7.4 \pm 0.67$ & $14.3 \pm 0.31$
\end{tabular}

Results:

$\begin{array}{lcc} & \mathbf{p H} & \mathbf{S O}_{2} \\ \mathrm{a} & 7.396 \pm 0.003 & 96.7 \pm 0.07 \\ \mathrm{sC} & 7.345 \pm 0.005 & 38.0 \pm 0.70 \\ \mathrm{D}(\mathrm{a}-\mathrm{sC}) & 0.051 \pm 0.002 & 58.7 \pm 0.73 \\ \mathrm{a} & 7.391 \pm 0.004 & 96.7 \pm 0.10 \\ \mathrm{vJ} & 7.353 \pm 0.003 & 68.2 \pm 0.74 \\ \mathrm{D}(\mathrm{a}-\mathrm{vJ}) & 0.038 \pm 0.002 & 28.5 \pm 0.74 \\ \mathrm{a} & 7.385 \pm 0.003 & 96.8 \pm 0.13 \\ \mathrm{vH} & 7.360 \pm 0.003 & 76.8 \pm 0.62 \\ \mathrm{D}(\mathrm{a}-\mathrm{vH}) & 0.025 \pm 0.002 & 20.0 \pm 0.58 \\ \mathrm{a} & 7.395 \pm 0.003 & 96.3 \pm 0.10 \\ \mathrm{aP} & 7.372 \pm 0.003 & 76.8 \pm 0.42 \\ \mathrm{D}(\mathrm{a}-\mathrm{aP}) & 0.023 \pm 0.001 & 19.5 \pm 0.41 \\ \mathrm{a} & 7.395 \pm 0.002 & 96.6 \pm 0.91 \\ \mathrm{vC} & 7.389 \pm 0.002 & 77.1 \pm 1.19 \\ \mathrm{D}(\mathrm{a}-\mathrm{aC}) & 0.015 \pm 0.002 & 19.6 \pm 0.73 \\ \mathrm{a} & 7.388 \pm 0.003 & 96.5 \pm 0.10 \\ \mathrm{vR} & 7.375 \pm 0.003 & 87.8 \pm 0.46 \\ \mathrm{D}(\mathrm{a}-\mathrm{vR}) & 0.013 \pm 0.001 & 8.8 \pm 0.40 \\ \mathrm{a} & 7.408 \pm 0.004 & - \\ \mathrm{L} & 7.324 \pm 0.004 & - \\ \mathrm{D}(\mathrm{a}-\mathrm{L}) & 0.084 \pm 0.007 & -\end{array}$

Discussion: The "normal" $\mathrm{pO}_{2}, \mathrm{SO}_{2}, \mathrm{pS}$ levels are: (1) maximal in plasma of $\mathrm{vR}$ blood and $\mathrm{L}$ (with $\mathrm{pO}_{2}>\mathrm{pCO}_{2}$ ); and (2) minimal in plasma of $\mathrm{sC}$ blood (with $\mathrm{pO}_{2}<\mathrm{pCO}_{2}$ ). In other regions, the levels are found between $\mathrm{vR}$ and maximal ones, where $\mathrm{pO}_{2}$ and $\mathrm{pCO}_{2}$ relationships can be different. The evaluation of $\mathrm{pO}_{2}$ and $\mathrm{pCO}_{2}$ relationships promotes more complete understanding of each organ's role in regulation of oxyhemoglobin regional dissociation process through their metabolism.

References

Gebel GY, Kruglov AG, et al: 7th World Cong. Int. Soc. Cardiothor. Surgery, Dusseldorf, Germany, 1997; 348

Gebel GY, Kruglov AG et al. 12th World Congr. Anaesthesiol, Montreal, Canada, 2000, p.14, 220, 284.

Key words: organs; partial pressures; plasma; respiratory gases; spinal fluid

Prebosp Disast Med 2001;16(2):s31 